Copyright (C 2014 IEEE. Personal use of this material is permitted. Permission from IEEE must be obtained for all other uses, in any current or future media, including reprinting/republishing this material for advertising or promotional purposes, creating new collective works, for resale or redistribution to servers or lists, or reuse of any copyrighted component of this work in other works. 


\section{Simplified MIMO Relay Design for Multicasting from Multiple-Sources}

\author{
Muhammad R. A. Khandaker \\ Dept. of Electronic and Electrical Engineering \\ University College London \\ Gower Street, London, WC1E 7JE, United Kingdom \\ Email:m.khandaker@ucl.ac.uk
}

\author{
Yue Rong \\ Dept. of Electrical and Computer Engineering \\ Curtin University \\ Bentley, WA, 6102, Australia \\ Email: y.rong@curtin.edu.au
}

\begin{abstract}
In this paper, we consider a dual-hop multicasting multiple-input multiple-output (MIMO) relay system where multiple transmitters multicast their own messages to a group of receivers with the aid of a relay node, and all nodes are equipped with multiple antennas. We aim at minimizing the maximal MSE of the signal waveform estimation among all receivers subjecting to power constraints at the transmitters and the relay node. We propose a low complexity solution for the problem under some mild approximation. In particular, we show that under (moderately) high signal-to-noise ratio (SNR) assumption, the min-max optimization problem can be solved using the semidefinite programming (SDP) technique. Numerical simulations demonstrate the effectiveness of the proposed algorithm.
\end{abstract}

\section{INTRODUCTION}

In many practical communication systems, multiple users (transmitters) need to send their messages to a group of receivers simultaneously. For example, in an online interactive gaming scenario, all the participants are interested to know the current status of their rivals. If the participants multicast their information, the interested users can receive all the messages simultaneously. Multicasting from multiple sources can also be used to support video conferencing and webcasts among multiple users. Next generation wireless communication standards such as WiMAX $802.16 \mathrm{~m}$ and 3GPP LTE-Advanced have already included technologies which enable better multicasting solutions based on multi-antenna and beamforming techniques [1].

Due to its nonconvex nature, the problem of designing optimal beamforming vectors for multicasting is hard in general. The authors of [2] have designed transmit beamformers for physical layer multicasting using rank relaxations. It has been proven in [2] that the beamforming problem is NP-hard. Using lower complexity transmission schemes, the information theoretic capacity of the multi-antenna multicasting channel was studied in [3].

The above works [2]-[4] considered single-hop multicasting systems. However, as the transmitter-receiver distance increases, it becomes necessary to adopt relay nodes to efficiently combat the pathloss of wireless channel. The authors in [5] studied the lower-bound for the outage probability of cooperative multi-antenna multicasting schemes based on the amplify-and-forward (AF) strategy where the users are equipped with a single antenna. Joint transmit and relay precoding design problems were investigated in [6] for a twohop multicasting MIMO relay system where all nodes are equipped with multiple antennas. An iterative algorithm has been developed in [6] to jointly optimize the source, relay, and receiver matrices. In order to reduce the computational complexity of the iterative algorithm, a simplified algorithm has also been proposed in [6] for the two-hop multicasting system.

In this paper, we consider dual-hop multicasting MIMO relay systems where multiple transmitters multicast their messages to a group of receivers with the aid of a relay node. The transmitters, relay node, and receivers are all equipped with multiple antennas. To the best of our knowledge, such multicasting (from multiple sources) MIMO relay system has not been investigated in existing works. Note that our paper generalizes the multicasting scheme in [6] by multicasting from multiple sources instead of the single-transmitter multicasting in [6]. It is obvious that due to the introduction of multiple users, the mean-squared error (MSE) matrix decomposition and hence the source and relay matrices optimization procedure become much more challenging than that for the single-transmitter systems. We aim at minimizing the maximal MSE of the signal waveform estimation among all receivers subjecting to power constraints at the transmitters and the relay node. The problem is highly nonconvex with matrix variables and the exactly optimal solution is very difficult to obtain. By exploiting the optimal structure of the relay precoding matrices, we propose a low-complexity solution to the problem under some mild approximation. In particular, we show that under (moderately) high SNR assumption, the problem can be solved using standard semidefinite programming (SDP) techniques. Numerical simulations demonstrate the effectiveness of the proposed algorithm. Note that the proposed algorithm supports multicasting multiple data streams in contrast to the existing single data stream multicasting schemes [1]-[5].

\section{System Model}

We consider a multicasting MIMO relay system where $K$ transmitters simultaneously multicast their information to $M$ receivers with the aid of a relay node as illustrated in Fig. 1 . The $k$ th transmitter and the relay node are equipped with 
$N_{\mathrm{s}, k}$ and $N_{\mathrm{r}}$ antennas, respectively. For the sake of notational simplicity, we assume that each receiver has $N_{\mathrm{d}}$ antennas. The algorithm developed in this paper can be straightforwardly extended to multicasting systems where receivers have different number of antennas. The direct links between the transmitters and the receivers are not considered since we assume that these direct links undergo much larger path attenuations compared with the links via the relay node.

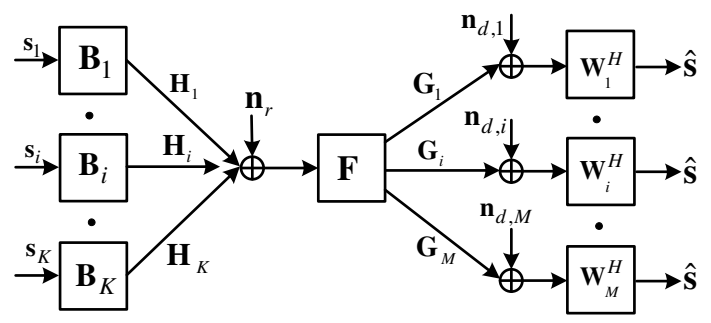

Fig. 1. Block diagram of a dual-hop multiuser multicasting MIMO relay system.

We assume that the relay node works in half-duplex mode. Thus the communication between the transmitters and receivers is accomplished in two time slots. In the first time slot, the $k$ th transmitter linearly precodes an $N_{\mathrm{s}, k} \times 1$ modulated signal vector $\mathbf{s}_{k}$ (common message to all receivers) by an $N_{\mathrm{s}, k} \times N_{\mathrm{s}, k}$ precoding matrix $\mathbf{B}_{k}$ and transmits the precoded vector $\mathbf{B}_{k} \mathbf{s}_{k}$ to the relay node. We assume that $\mathrm{E}\left[\mathbf{s}_{k} \mathbf{s}_{k}^{H}\right]=\mathbf{I}_{N_{\mathrm{s}, k}}$, where $\mathrm{E}[\cdot]$ denotes statistical expectation, $(\cdot)^{H}$ stands for the matrix Hermitian transpose, and $\mathbf{I}_{n}$ is an $n \times n$ identity matrix. We denote $N_{\mathrm{b}}=\sum_{k=1}^{K} N_{\mathrm{s}, k}$ as the total number of independent data streams from all transmitters. The received signal vector at the relay node is given by

$$
\mathbf{y}_{\mathrm{r}}=\sum_{k=1}^{K} \mathbf{H}_{k} \mathbf{B}_{k} \mathbf{s}_{k}+\mathbf{n}_{\mathrm{r}} \triangleq \mathbf{H B} \mathbf{s}+\mathbf{n}_{\mathrm{r}}
$$

where $\mathbf{H}_{k}$ is the $N_{\mathrm{r}} \times N_{\mathrm{s}, k}$ MIMO channel matrix between the $k$ th transmitter and the relay node, $\mathbf{y}_{\mathrm{r}}$ and $\mathbf{n}_{\mathrm{r}}$ are the $N_{\mathrm{r}} \times 1$ received signal and additive Gaussian noise vectors introduced at the first relay node, respectively, $\mathbf{H}=\left[\mathbf{H}_{1}, \cdots, \mathbf{H}_{K}\right]$, and $\mathbf{B} \triangleq \operatorname{bd}\left(\mathbf{B}_{1}, \cdots, \mathbf{B}_{K}\right), \mathbf{s} \triangleq\left[\mathbf{s}_{1}^{T}, \cdots, \mathbf{s}_{K}^{T}\right]^{T}$. Here $\operatorname{bd}(\cdot)$ stands for a block diagonal matrix and $(\cdot)^{T}$ denotes the matrix (vector) transpose.

In the second time slot, the transmitters remain silent and the relay node multiplies (linearly precodes) the received signal vector $\mathbf{y}_{\mathrm{r}}$ by an $N_{\mathrm{r}} \times N_{\mathrm{r}}$ relay precoding matrix $\mathbf{F}$ and multicasts the precoded signal vector

$$
\mathbf{x}_{\mathrm{r}}=\mathbf{F} \mathbf{y}_{\mathrm{r}}
$$

to all the $M$ destination nodes. From (1), (2), the received signal vector at the $i$ th receiver can be written as

$$
\begin{aligned}
\mathbf{y}_{\mathrm{d}, i} & =\mathbf{G}_{i} \mathbf{F}\left(\mathbf{H B} \mathbf{s}+\mathbf{n}_{\mathrm{r}}\right)+\mathbf{n}_{\mathrm{d}, i} \\
& \triangleq \overline{\mathbf{A}}_{i} \mathbf{s}+\overline{\mathbf{n}}_{i}, \quad i=1, \cdots, M
\end{aligned}
$$

where $\mathbf{G}_{i}$ is the $N_{\mathrm{d}} \times N_{\mathrm{r}}$ MIMO channel matrix between the relay node and the $i$ th receiver and $\mathbf{n}_{\mathrm{d}, i}$ is the additive Gaussian noise vector at the $i$ th receiver. In (3), $\overline{\mathbf{A}}_{i} \triangleq \mathbf{G}_{i} \mathbf{F H B}$ is the equivalent MIMO channel between the transmitters and the $i$ th receiver, and $\overline{\mathbf{n}}_{i} \triangleq \mathbf{G}_{i} \mathbf{F} \mathbf{n}_{\mathrm{r}}+\mathbf{n}_{\mathrm{d}, i}$ is the equivalent noise vector at the $i$ th receiver. We assume that all noises are independent and identically distributed (i.i.d.) complex circularly symmetric Gaussian noise with zero mean and unit variance. Thus the covariance matrix of $\overline{\mathbf{n}}_{i}, \overline{\mathbf{C}}_{i}=\mathrm{E}\left[\overline{\mathbf{n}}_{i} \overline{\mathbf{n}}_{i}^{H}\right]$ is given by $\overline{\mathbf{C}}_{i}=\mathbf{G}_{i} \mathbf{F} \mathbf{F}^{H} \mathbf{G}_{i}^{H}+\mathbf{I}_{N_{\mathrm{d}}}, i=1, \cdots, M$.

In the next section, we consider optimizing the source and relay precoding matrices in order to improve the MSE performance of the system.

\section{Proposed Source And Relay Design Algorithm}

Due to its simplicity, a linear receiver is used at each receiver to retrieve the transmitted signals. Denoting $\mathbf{W}_{i}$ as an $N_{\mathrm{d}} \times N_{\mathrm{b}}$ weight matrix at the $i$ th receiver, the estimated signal vector $\hat{\mathbf{s}}_{i}$ is given by

$$
\hat{\mathbf{s}}_{i}=\mathbf{W}_{i}^{H} \mathbf{y}_{\mathrm{d}, i}, \quad i=1, \cdots, M .
$$

From (4), the MSE of the signal waveform estimation at the $i$ th receiver is given by

$$
E_{i}=\operatorname{tr}\left(\left(\mathbf{W}_{i}^{H} \overline{\mathbf{A}}_{i}-\mathbf{I}_{N_{\mathrm{b}}}\right)\left(\mathbf{W}_{i}^{H} \overline{\mathbf{A}}_{i}-\mathbf{I}_{N_{\mathrm{b}}}\right)^{H}+\mathbf{W}_{i}^{H} \overline{\mathbf{C}}_{i} \mathbf{W}_{i}\right)(5)
$$

where $\operatorname{tr}(\cdot)$ denotes matrix trace. Obviously, the power consumed by the $k$ th transmitter is $\operatorname{tr}\left(\mathbf{B}_{k} \mathbf{B}_{k}^{H}\right), k=1, \cdots, K$. And from (2), the transmission power consumed by the relay node is given by

$$
\operatorname{tr}\left(\mathrm{E}\left[\mathbf{x}_{\mathrm{r}} \mathbf{x}_{\mathrm{r}}^{H}\right]\right)=\operatorname{tr}\left(\mathbf{F}\left(\mathbf{H B B}^{H} \mathbf{H}^{H}+\mathbf{I}_{N_{\mathrm{r}}}\right) \mathbf{F}^{H}\right) .
$$

Given the transmission power constraints at the transmitters and the relay node, we aim at minimizing the maximal MSE of the signal waveform estimations among all receivers. This problem formulation is important when the power consumption is a strict system constraint that cannot be relaxed. In this case, the transmit, relay, and receive matrices optimization problem can be formulated as

$$
\begin{aligned}
\min _{\left\{\mathbf{B}_{k}\right\}, \mathbf{F},\left\{\mathbf{W}_{i}\right\}} & \max _{i} E_{i} \\
\text { s.t. } & \operatorname{tr}\left(\mathbf{F}\left(\mathbf{H B B} \mathbf{B}^{H} \mathbf{H}^{H}+\mathbf{I}_{N_{\mathrm{r}}}\right) \mathbf{F}^{H}\right) \leq P_{\mathrm{r}} \\
& \operatorname{tr}\left(\mathbf{B}_{k} \mathbf{B}_{k}^{H}\right) \leq P_{\mathrm{s}, k}, k=1, \cdots, K
\end{aligned}
$$

where $\left\{\mathbf{W}_{i}\right\} \triangleq\left\{\mathbf{W}_{i}, i=1, \cdots, M\right\},\left\{\mathbf{B}_{k}\right\} \triangleq\left\{\mathbf{B}_{k}, k=\right.$ $1, \cdots, K\},(7 \mathrm{~b})$ and (7c) are the transmission power constraints at the relay node and the $k$ th transmitter, respectively, and $P_{\mathrm{r}}>0, P_{\mathrm{s}, k}>0$ are the corresponding power budgets.

For any given $\left\{\mathbf{B}_{k}\right\}$ and $\mathbf{F}$, the receiver $\mathbf{W}_{i}$ minimizing $E_{i}$ in (5) is the linear minimal mean-squared error (MMSE) filter [8] and given by

$$
\mathbf{W}_{i}=\left(\overline{\mathbf{A}}_{i} \overline{\mathbf{A}}_{i}^{H}+\overline{\mathbf{C}}_{i}\right)^{-1} \overline{\mathbf{A}}_{i}, \quad i=1, \cdots, M
$$

where $(\cdot)^{-1}$ denotes matrix inversion. By substituting (8) back into (5), we have

$$
E_{i}=\operatorname{tr}\left(\left[\mathbf{I}_{N_{\mathrm{b}}}+\overline{\mathbf{A}}_{i}^{H} \overline{\mathbf{C}}_{i}^{-1} \overline{\mathbf{A}}_{i}\right]^{-1}\right), \quad i=1, \cdots, M .
$$


Therefore, we can equivalently rewrite the problem (7) as

$$
\begin{aligned}
\min _{\left\{\mathbf{B}_{k}\right\}, \mathbf{F}} & \max _{i} \operatorname{tr}\left(\left[\mathbf{I}_{N_{\mathrm{b}}}+\overline{\mathbf{A}}_{i}^{H} \overline{\mathbf{C}}_{i}^{-1} \overline{\mathbf{A}}_{i}\right]^{-1}\right) \\
\text { s.t. } & \operatorname{tr}\left(\mathbf{F}\left(\mathbf{H B B}^{H} \mathbf{H}^{H}+\mathbf{I}_{N_{\mathrm{r}}}\right) \mathbf{F}^{H}\right) \leq P_{\mathrm{r}} \\
& \operatorname{tr}\left(\mathbf{B}_{k} \mathbf{B}_{k}^{H}\right) \leq P_{\mathrm{s}, k}, k=1, \cdots, K .
\end{aligned}
$$

The min-max problem (10) is highly nonconvex with matrix variables, and an exactly optimal solution is very hard to obtain with a reasonable computational complexity. In the following, we propose a low complexity solution to the problem (10).

It can be shown similar to [9] that the optimal $\mathbf{F}$ for each link with the input-output relationship given by (3) has the generic structure of

$$
\mathbf{F}=\mathbf{T D}^{H}
$$

where $\mathbf{D}=\left(\mathbf{H B B}^{H} \mathbf{H}^{H}+\mathbf{I}_{N_{\mathbf{r}}}\right)^{-1} \mathbf{H B}$ is the weight matrix of the linear MMSE filter for the received signal vector at the relay node given by (1), and the linear filter $\mathbf{T}$ can be treated as the transmit precoding matrix for the effective second-hop MIMO multicasting channel, which will be designed later.

Using the optimal $\mathbf{F}$ in (11), the MSE of the signal waveform estimation at the $i$ th receiver in (9) can be equivalently decomposed to

$$
\begin{aligned}
E_{i}= & \operatorname{tr}\left(\left[\mathbf{I}_{N_{\mathrm{b}}}+\mathbf{B}^{H} \mathbf{H}^{H} \mathbf{H B}\right]^{-1}\right) \\
& +\operatorname{tr}\left(\left[\mathbf{R}^{-1}+\mathbf{T}^{H} \mathbf{G}_{i}^{H} \mathbf{G}_{i} \mathbf{T}\right]^{-1}\right), i=1, \cdots, M(
\end{aligned}
$$

where

$$
\mathbf{R}=\mathbf{B}^{H} \mathbf{H}^{H}\left(\mathbf{H B B}^{H} \mathbf{H}^{H}+\mathbf{I}_{N_{\mathrm{r}}}\right)^{-1} \mathbf{H B} .
$$

Note that the first term in (12) $\operatorname{tr}\left(\left[\mathbf{I}_{N_{\mathrm{b}}}+\mathbf{B}^{H} \mathbf{H}^{H} \mathbf{H B}\right]^{-1}\right)$ is actually the MSE of estimating the signal vector $\mathbf{s}$ from the received signal vector (1) at the relay node using the linear MMSE receiver $\mathbf{D}$, while the remaining term in (12) can be viewed as the increment of the MSE introduced by the second-hop. Using the optimal structure of $\mathbf{F}$ in (11), the transmission power consumed by the relay node can be rewritten as $\operatorname{tr}\left(\mathbf{T R T}^{H}\right)$. And the first term in (12) can be rewritten as $\operatorname{tr}\left(\left[\mathbf{I}_{N_{\mathrm{r}}}+\sum_{k=1}^{K} \mathbf{H}_{k} \mathbf{P}_{k} \mathbf{H}_{k}^{H}\right]^{-1}\right)+N_{\mathrm{b}}-N_{\mathrm{r}}$ where $\mathbf{P}_{k}=\mathbf{B}_{k} \mathbf{B}_{k}^{H}, k=1, \cdots, K$, is the covariance matrix of the signal transmitted by the $k$ th user. Therefore, the problem (10) can be equivalently rewritten as

$$
\begin{array}{rc}
\min _{\left\{\mathbf{P}_{k}\right\}, \mathbf{T}} \max _{i} \operatorname{tr}\left(\left[\mathbf{I}_{N_{\mathrm{r}}}+\sum_{k=1}^{K} \mathbf{H}_{k} \mathbf{P}_{k} \mathbf{H}_{k}^{H}\right]^{-1}\right) \\
\\
\quad+\operatorname{tr}\left(\left[\mathbf{R}^{-1}+\mathbf{T}^{H} \mathbf{G}_{i}^{H} \mathbf{G}_{i} \mathbf{T}\right]^{-1}\right) \\
\text { s.t. } & \operatorname{tr}\left(\mathbf{T R T} \mathbf{T}^{H}\right) \leq P_{\mathrm{r}} \\
& \operatorname{tr}\left(\mathbf{P}_{k}\right) \leq P_{\mathrm{s}, k}, \quad \mathbf{P}_{k} \succcurlyeq 0, k=1, \cdots, K
\end{array}
$$

where $\mathbf{A} \succcurlyeq 0$ indicates that matrix $\mathbf{A}$ is positive semidefinite (PSD).

By applying the matrix inversion lemma, matrix $\mathbf{R}$ in (13) can be rewritten as

$$
\mathbf{R}=\mathbf{B}^{H} \mathbf{H}^{H} \mathbf{H B}\left(\mathbf{B}^{H} \mathbf{H}^{H} \mathbf{H B}+\mathbf{I}_{N_{\mathrm{b}}}\right)^{-1} .
$$

An interesting observation from (15) is that with increasing first-hop SNR, the term $\mathbf{B}^{H} \mathbf{H}^{H} \mathbf{H B}$ approaches infinity. And at a (moderately) high SNR level, there is $\mathbf{B}^{H} \mathbf{H}^{H} \mathbf{H B} \gg \mathbf{I}_{N_{\mathrm{b}}}$. Here for matrices $\mathbf{X}$ and $\mathbf{Y}, \mathbf{X} \gg \mathbf{Y}$ indicates that the eigenvalues of $(\mathbf{X}-\mathbf{Y})$ are much greater than zero. Thus, we can approximate $\mathbf{R}$ as $\mathbf{I}_{N_{\mathrm{b}}}$ for the high SNR case [10]. As a consequence, $\operatorname{tr}\left(\left[\mathbf{R}^{-1}+\mathbf{T}^{H} \mathbf{G}_{i}^{H} \mathbf{G}_{i} \mathbf{T}\right]^{-1}\right)$ in (14a) can be closely upper-bounded by $\operatorname{tr}\left(\left[\mathbf{I}_{N_{\mathrm{b}}}+\mathbf{T}^{H} \mathbf{G}_{i}^{H} \mathbf{G}_{i} \mathbf{T}\right]^{-1}\right)$, $i=1, \cdots, M$, and the tightness of these bounds increase with the increase in SNR. Therefore, the problem (14) can be approximated as

$$
\begin{aligned}
& \min _{\left\{\mathbf{P}_{k}\right\}, \mathbf{T}} \max _{i} \operatorname{tr}\left(\left[\mathbf{I}_{N_{\mathrm{r}}}+\sum_{k=1}^{K} \mathbf{H}_{k} \mathbf{P}_{k} \mathbf{H}_{k}^{H}\right]^{-1}\right) \\
&+\operatorname{tr}\left(\left[\mathbf{I}_{N_{\mathrm{b}}}+\mathbf{T}^{H} \mathbf{G}_{i}^{H} \mathbf{G}_{i} \mathbf{T}\right]^{-1}\right) \\
& \text { s.t. } \operatorname{tr}\left(\mathbf{T} \mathbf{T}^{H}\right) \leq P_{\mathrm{r}} \\
& \operatorname{tr}\left(\mathbf{P}_{k}\right) \leq P_{\mathrm{s}, k}, \quad \mathbf{P}_{k} \succcurlyeq 0, k=1, \cdots, K
\end{aligned}
$$

Interestingly, it can be seen from the problem (16) that $\mathbf{T}$ does not affect the first term of the objective function (16a) and $\mathbf{P}_{k}, k=1, \cdots, K$, are irrelevant to the remaining terms of (16a). This fact implies that the objective function (16a) and the constraints (16b) and (16c) are decoupled with respect to the optimization variables $\left\{\mathbf{P}_{k}\right\}$ and $\mathbf{T}$. In this case, matrices $\left\{\mathbf{P}_{k}\right\}$ can be determined independent of $\mathbf{T}$, and vice-versa, which greatly simplifies the design of the transmit and relay matrices. Therefore, with the (relatively) high SNR assumption, the problem (16) can be decomposed into the following source covariance matrices optimization problem

$$
\begin{array}{ll}
\min _{\left\{\mathbf{P}_{k}\right\}} & \operatorname{tr}\left(\left[\mathbf{I}_{N_{1}}+\sum_{k=1}^{K} \mathbf{H}_{1, k} \mathbf{P}_{k} \mathbf{H}_{1, k}^{H}\right]^{-1}\right) \\
\text { s.t. } & \operatorname{tr}\left(\mathbf{P}_{k}\right) \leq P_{\mathrm{s}, k}, \quad \mathbf{P}_{k} \succcurlyeq 0, k=1, \cdots, K
\end{array}
$$

and the relay precoder optimization problem

$$
\begin{array}{ll}
\min _{\mathbf{T}} & \max _{i} \operatorname{tr}\left(\left[\mathbf{I}_{N_{\mathrm{b}}}+\mathbf{T}^{H} \mathbf{G}_{i}^{H} \mathbf{G}_{i} \mathbf{T}\right]^{-1}\right) \\
\text { s.t. } & \operatorname{tr}\left(\mathbf{T} \mathbf{T}^{H}\right) \leq P_{\mathrm{r} .}
\end{array}
$$

The source covariance matrices optimization problem (17) can be solved as follows. By introducing a PSD matrix $\mathbf{X}$ with $\mathbf{X} \succcurlyeq\left[\mathbf{I}_{N_{1}}+\sum_{k=1}^{K} \mathbf{H}_{1, k} \mathbf{P}_{k} \mathbf{H}_{1, k}^{H}\right]^{-1}$, where $\mathbf{A} \succcurlyeq \mathbf{B}$ means that $\mathbf{A}-\mathbf{B} \succcurlyeq 0$, and using the Schur complement [11], the problem (17) can be converted to the problem of

$$
\begin{aligned}
\min _{\left\{\mathbf{P}_{k}\right\}, \mathbf{X}} & \operatorname{tr}(\mathbf{X}) \\
\text { s.t. } & \left(\begin{array}{cc}
\mathbf{X} & \mathbf{I}_{N_{\mathrm{r}}} \\
\mathbf{I}_{N_{\mathrm{r}}} & \mathbf{I}_{N_{\mathrm{r}}}+\sum_{k=1}^{K} \mathbf{H}_{1, k} \mathbf{P}_{k} \mathbf{H}_{1, k}^{H}
\end{array}\right) \succcurlyeq 0 \\
& \operatorname{tr}\left(\mathbf{P}_{k}\right) \leq P_{\mathrm{s}, k}, \quad \mathbf{P}_{k} \succcurlyeq 0, k=1, \cdots, K .
\end{aligned}
$$

The problem (19) is a convex SDP problem which can be efficiently solved by the disciplined convex programming toolbox CVX [12], where interior-point method-based solvers such as 
SeDuMi or SDPT3 are called internally, at a complexity order of $\mathcal{O}\left(\left(\sum_{k=1}^{K} N_{\mathrm{s}, k}^{2}+K\right)^{3.5}\right)$ [13].

By introducing $\mathbf{T} \mathbf{T}^{H} \triangleq \mathbf{Q}$, the problem (18) can be equivalently rewritten as

$$
\begin{array}{ll}
\min _{\mathbf{Q}} & \max _{i} \operatorname{tr}\left(\left[\mathbf{I}_{N_{\mathrm{d}}}+\mathbf{G}_{i} \mathbf{Q G}_{i}^{H}\right]^{-1}\right)+N_{\mathrm{b}}-N_{\mathrm{d}} \\
\text { s.t. } & \operatorname{tr}(\mathbf{Q}) \leq P_{\mathrm{r}}, \quad \mathbf{Q} \succcurlyeq 0 .
\end{array}
$$

By introducing $\mathbf{Y}_{i} \succcurlyeq\left[\mathbf{I}_{N_{\mathrm{d}}}+\mathbf{H}_{L, i} \mathbf{Q} \mathbf{H}_{L, i}^{H}\right]^{-1}, i=1, \cdots, M$, and a real-valued slack variable $t$, the problem (20) can be equivalently transformed to

$$
\begin{aligned}
& \min _{t, \mathbf{Q},\left\{\mathbf{Y}_{i}\right\}} t \\
& \text { s.t. } \operatorname{tr}\left(\mathbf{Y}_{i}\right) \leq t, \quad i=1, \cdots, M \\
& \operatorname{tr}(\mathbf{Q}) \leq P_{\mathrm{r}} \\
& \left(\begin{array}{cc}
\mathbf{Y}_{i} & \mathbf{I}_{N_{\mathrm{d}}} \\
\mathbf{I}_{N_{\mathrm{d}}} & \mathbf{I}_{N_{\mathrm{d}}}+\mathbf{G}_{i} \mathbf{Q} \mathbf{G}_{i}^{H}
\end{array}\right) \succcurlyeq 0, i=1, \cdots, M(21 \mathrm{~d}) \\
& t \geq 0, \quad \mathbf{Q} \succcurlyeq 0
\end{aligned}
$$

where $\left\{\mathbf{Y}_{i}\right\} \triangleq\left\{\mathbf{Y}_{i}, i=1, \cdots, M\right\}$ and we use the Schur complement to obtain (21d). Note that in the above formulation, $t$ provides an MSE upper-bound for the secondhop channels. The problem (21) is an SDP problem which can be efficiently solved by the disciplined convex programming toolbox CVX [12] at a maximal complexity order of $\mathcal{O}\left(\left(N_{\mathrm{r}}^{2}+M+1\right)^{3.5}\right)[13]$.

\section{NUMERICAL EXAMPLES}

In this section, we study the performance of the proposed multi-source multicasting MIMO relay optimization algorithm through numerical simulations. For simplicity, we assume that the transmitters and the receivers are equipped with $N_{\mathrm{S}}$ and $N_{\mathrm{d}}$ antennas each, respectively, and $P_{\mathrm{s}, k}=P_{\mathrm{s}}, k=1, \cdots, K$. We simulate a flat Rayleigh fading environment where the channel matrices have entries with zero mean and variances $1 / N_{\mathrm{s}}$ and $1 / N_{\mathrm{r}}$, for $\mathbf{H}_{k}, k=1, \cdots, K$, and $\mathbf{G}_{i}, i=1, \cdots, M$, respectively. All simulation results are averaged over 500 independent channel realizations.

Since there is no existing solution for the problem addressed in this paper, we compare the performance of the proposed min-max MSE algorithm in Section III with the naive amplifyand-forward (NAF) algorithm in terms of both MSE and BER. For the NAF scheme, we use $\mathbf{B}_{k}=\sqrt{P_{\mathrm{s}} / N_{\mathrm{s}}} \mathbf{I}_{N_{\mathrm{s}}}, k=$ $1, \cdots, K$, and $\mathbf{F}=\sqrt{P_{\mathrm{r}} / \operatorname{tr}\left(\mathbf{H B B}^{H} \mathbf{H}^{H}+\mathbf{I}_{N_{\mathrm{r}}}\right)} \mathbf{I}_{N_{\mathrm{r}}}$.

In the first example, we compare the performance of the proposed algorithm with the NAF approach in terms of the MSE normalized by the number of data streams (NMSE) for $K=2, M=4, N_{\mathrm{s}}=2, N_{\mathrm{r}}=4$, and $N_{\mathrm{d}}=8$. Fig. 2 shows the MSE performance of both algorithms versus $P_{\mathrm{s}}$ with $P_{\mathrm{r}}=20 \mathrm{~dB}$. For the proposed algorithm, we plot the NMSE of the user with the worst channel (Worst) and the average of all the users (Avg.). Our results clearly demonstrate the better performance of the proposed joint transmitter and relay optimization algorithm. It can be seen that the proposed algorithm consistently yields the lowest average MSE over the entire $P_{\mathrm{s}}$ region.

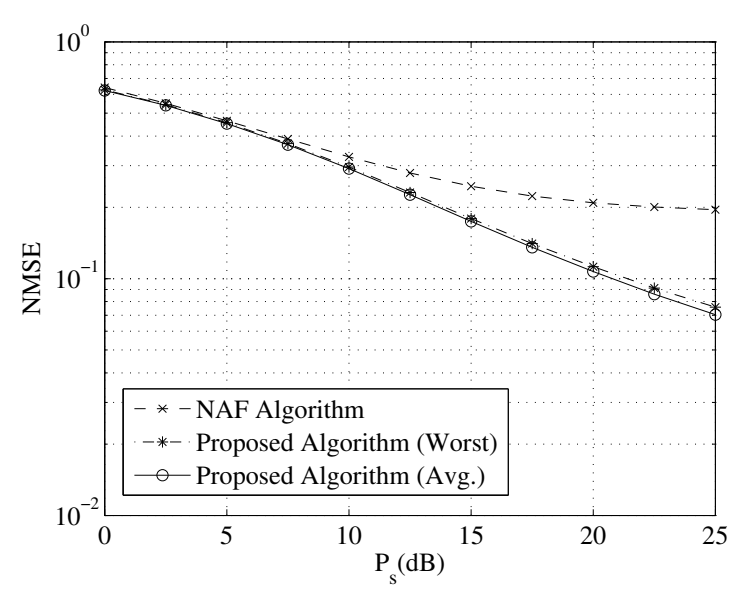

Fig. 2. Example 1: Normalized MSE versus $P_{\mathrm{S}} . K=2, M=4, N_{\mathrm{s}}=$ $2, N_{\mathrm{r}}=4, N_{\mathrm{d}}=8, P_{\mathrm{r}}=20 \mathrm{~dB}$.

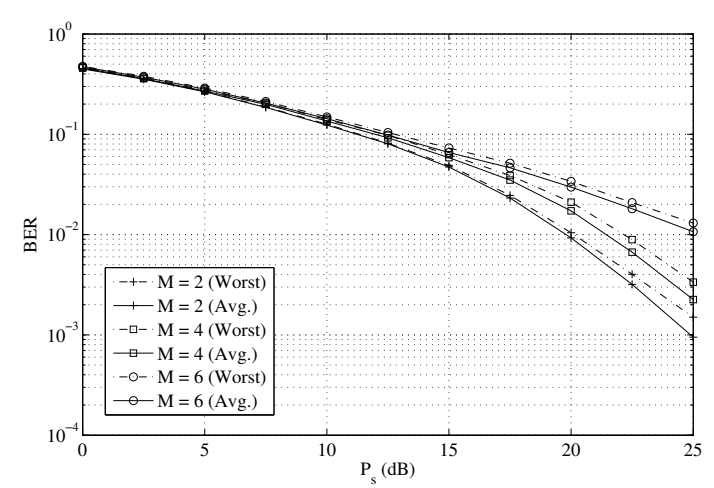

Fig. 3. Example 2: BER versus $P_{\mathrm{S}}$. Varying number of receivers, $K=2$, $N_{\mathrm{s}}=2, N_{\mathrm{r}}=4, N_{\mathrm{d}}=8, P_{\mathrm{r}}=20 \mathrm{~dB}$.

In the second example, we compare the BER performance of the proposed algorithm for different number of receivers. QPSK signal constellations are used to modulate the transmitted signals. This time we set $K=2, N_{\mathrm{s}}=2, N_{\mathrm{r}}=4$, and $N_{\mathrm{d}}=8$. Fig. 3 shows the BER of the proposed algorithm versus $P_{\mathrm{s}}$ with $P_{\mathrm{r}}=20 \mathrm{~dB}$ for different number of receivers. It can be clearly seen from Fig. 3 that as we increase the number of receivers, the worst-user BER keeps increasing which is analogous to the results obtained in [6]. This is reasonable since it is more likely to find a worse relay-receiver channel among the increased number of users and we choose the worstuser MSE as the objective function.

\section{CONCLUSIONS}

We considered a dual-hop multicasting MIMO relay system with multi-antenna nodes and proposed transmit and relay precoding matrices based on the min-max MSE criterion. Under some mild approximation, we show that the problem can be solved with a significantly lower computational complexity. Simulation results demonstrate that the proposed transmitter and relay design algorithm outperforms the existing techniques. 


\section{REFERENCES}

[1] M. A. Khojastepour, A. Salehi-Golsefidi, and S. Rangarajan, "Towards an optimal beamforming algorithm for physical layer multicasting," in Proc. IEEE Inf. Theory Workshop, Oct. 16-20, 2011, pp. 395-399.

[2] N. D. Sidiropoulos, T. N. Davidson, and Z.-Q. Luo, "Transmit beamforming for physical-layer multicasting," IEEE Trans. Signal Process. vol. 54, pp. 2239-2251, Jun. 2006.

[3] N. Jindal and Z.-Q. Luo, "Capacity limits of multiple antenna multicast," in Proc. IEEE ISIT, Seattle, USA, Jul. 9-14, 2006, pp. 1841-1845.

[4] Q. Li and W.-K. Ma, "Multicast secrecy rate maximization for MISO channels with multiple multi-antenna eavesdroppers," in Proc. IEEE ICC, Kyoto, Japan, Jun. 5-9, 2011.

[5] H. Zhang, X. You, G. Wu, and H. Wang, "Cooperative multi-antenna multicasting for wireless networks," in IEEE GLOBECOM, Miami, FL, USA, Dec. 6-10, 2010

[6] M. R. A. Khandaker and Y. Rong, "Precoding design for MIMO relay multicasting," IEEE Trans. Wireless Commun., vol. 12, pp. 3544-3555, Jul. 2013.
[7] D. P. Palomar and Y. Jiang, MIMO Transceiver Design via Majorization Theory. now Publishers, 2007.

[8] S. M. Kay, Fundamentals of Statistical Signal Processing: Estimation Theory. Englewood Cilffs, NJ: Prentice Hall, 1993.

[9] Y. Rong, "Simplified algorithms for optimizing multiuser multi-hop MIMO relay systems," IEEE Trans. Commun., vol. 59, pp. 2896-2904, Oct. 2011.

[10] C. Song, K.-J. Lee, and I. Lee, "MMSE based transceiver designs in closed-loop non-regenerative MIMO relaying systems," IEEE Trans. Wireless Commun., vol. 9, pp. 2310-2319, Jul. 2010.

[11] S. Boyd and L. Vandenberghe, Convex Optimization. Cambridge, U. K. Cambridge University Press, 2004.

[12] M. Grant and S. Boyd, "Cvx: Matlab software for disciplined convex programming (web page and software)." http://cvxr.com/cvx, April, 2010.

[13] Y. Nesterov and A. Nemirovski, Interior Point Polynomial Algorithms in Convex Programming. Philadelphia, PA: SIAM, 1994. 